\title{
Spatiotemporal single-cell analysis reveals critical roles of mechano-sensing genes at the border zone in remodeling after myocardial infarction
}

\section{Shintaro Yamada}

Tokyo University https://orcid.org/0000-0002-3240-358X

Toshiyuki Ko

The University of Tokyo

Satoshi Hatsuse

The University of Tokyo

Seitaro Nomura

The University of Tokyo

Bo Zhang

The University of Tokyo

Zhehao Dai

The University of Tokyo

https://orcid.org/0000-0002-0363-7563

Shunsuke Inoue

The University of Tokyo

Masayuki Kubota

The University of Tokyo

Kosuke Sawami

The University of Tokyo

Takanobu Yamada

The University of Tokyo

Tatsuro Sassa

The University of Tokyo

Mikako Katagiri

The University of Tokyo

Kanna Fujita

The University of Tokyo

Manami Katoh

The University of Tokyo

Masamichi Ito

The University of Tokyo

Mutsuo Harada 
The University of Tokyo

\section{Haruhiro Toko}

The University of Tokyo

\section{Norifumi Takeda}

The University of Tokyo https://orcid.org/0000-0003-4818-3347

\section{Hiroyuki Morita}

The University of Tokyo

\section{Hiroyuki Aburatani}

The University of Tokyo

Issei Komuro ( $\nabla$ komuro-tky@umin.ac.jp )

The University of Tokyo Graduate School of Medicine

\section{Article}

Keywords: spatiotemporal single-cell analysis, myocardial infarction, left ventricular remodeling.

Posted Date: June 22nd, 2021

DOl: https://doi.org/10.21203/rs.3.rs-620498/v1

License: (1) (1) This work is licensed under a Creative Commons Attribution 4.0 International License. Read Full License

Version of Record: A version of this preprint was published at Nature Cardiovascular Research on November 17th, 2022. See the published version at https://doi.org/10.1038/s44161-022-00140-7. 


\section{Abstract}

The left ventricular remodeling after myocardial infarction (MI) causes heart failure, but its underlying mechanisms remain largely unknown. Here, we performed an integrative analysis of spatial transcriptomics and single cell RNA-seq in murine MI model and found that mechanical stress-response genes are expressed at the border zone and play a critical role in left ventricular remodeling after $\mathrm{Ml}$. Single-cardiomyocyte RNA-seq of the heart after MI divided cardiomyocytes into 8 clusters. We identified the cell cluster which was specifically derived from the ischemic area in an early stage after $\mathrm{Ml}$ and expressed mechano-sensing genes such as Csrp3, Ankrd1 and Ankrd2. Spatial transcriptomic analysis demonstrated that cardiomyocytes with this gene expression profile were specifically localized at the border zone in post-MI day 1. AAV9-mediated gene silencing of Csrp3 exacerbated cardiac dilatation and dysfunction after MI. Collectively, our datasets and findings not only provide an insight into spatiotemporal molecular pathogenesis of myocardial infarction but also highlight mechano-sensing genes at the border zone as an adaptive regulator of left ventricular remodeling.

\section{Introduction}

Despite decades of intensive research and therapeutic development, ischemic heart diseases are still a leading cause of death worldwide. Myocardial infarction (MI) causes various complications such as arrhythmia, valvular disease and heart failure ${ }^{1}$. In particular, the number of patients with heart failure has recently been increasing and becoming a more serious problem in many countries ${ }^{2,3}$. MI induces changes of left ventricular (LV) size, shape and function which were known as LV remodeling, leading to the development of heart failure ${ }^{4-6}$. Therefore, it is an urgent need to clarify molecular mechanisms of LV remodeling after $\mathrm{Ml}$ to inhibit the development of heart failure and improve the prognosis of patients with $\mathrm{MI}^{7-10}$. However, cellular and molecular behaviors change spatially and temporally after $\mathrm{Ml}$, it is difficult to precisely understand the molecular mechanisms of LV remodeling.

The infarcted heart is usually roughly divided into three zones such as an infarct zone (IZ), an ischemic border zone (BZ; defined as the sectors immediately adjacent to IZ) and a remote zone (RZ) ${ }^{11}$. Although previous studies showed transcriptional differences between myocardium proximal and distal to the $\mathrm{IZ}^{11-13}$, the precise characteristics of cardiomyocytes in each region have not been well explored. Furthermore, previous studies examined gene expressions using mRNA isolated from bulk samples and did not separate cardiomyocytes and non-cardiomyocytes. Here, we identified and characterized the distinctive transcriptional properties in each region of infarcted hearts by integrative analysis of singlecardiomyocyte RNA-seq and spatial transcriptomics. We unveiled a novel molecular adaptation of cardiomyocytes in the BZ characterized by transcriptional activation of mechano-sensing genes such as Csrp3 (also known as MLP, muscle LIM protein), Ankrd1 and Ankrd2.

\section{Results}


We made a murine $\mathrm{MI}$ model by ligating the left anterior descending artery. We separately isolated cardiomyocytes from ischemic region (IR, area including infarct zone and $2 \mathrm{~mm}$ of its lateral margin) and remote region (RR, area other than IR) and conducted single-cell RNA sequencing (scRNA-seq) and spatial transcriptomic analysis by Visium (10X Genomics) (Fig. 1a). We sequenced 775 cardiomyocytes from 7 murine hearts and analyzed 761 cardiomyocytes (sham, 88 cardiomyocytes; IR in post MI (pMI) day 1, 169 cardiomyocytes; RR in pMI day 1, 237 cardiomyocytes; IR in pMI day 7, 151 cardiomyocytes; RR in pMI day 7, 130 cardiomyocytes) in which $>2500$ genes were detected. Using clustering analysis for scRNA-seq data, cardiomyocytes derived from five different conditions (sham, pMI day 1 IR, pMI day 1 RR, pMI day 7 IR and pMI day 7 RR) were classified into eight cell clusters (Fig. 1b). We statistically analyzed the proportion of each cluster and found that there were some clusters only appeared in the specific conditions (Fig. 1c). For example, cluster 6 accounts for the majority of sham cardiomyocytes and highly expressed genes associated with mitochondrial functions and cardiomyocyte metabolism (Fig. 1c-e). By contrast, cluster 0 and cluster 1, mainly derived from cardiomyocytes of pMI day 1 and day 7, expressed genes associated with collagen production and inflammatory responses (inflammatory cytokine production), respectively (Fig. 1c-e). Although the proportions of many clusters were very similar between IR and RR, only cluster 7 was specifically derived from the cardiomyocytes of IR at pMI day 1 (Fig. 1c). Differential gene expression analysis showed that one of the key features in the cluster 7 was high expression of genes encoding for mechano-sensing proteins such as Csrp3, Ankrd1 and Ankrd2 (Fig. $1 \mathrm{~d}, \mathrm{e})$. The cluster 7 cardiomyocytes occupied about $30 \%$ of all cardiomyocytes in IR of pMI day 1 , but they were scarcely observed in other four conditions (Fig. 1c).

\section{Integrative analysis of spatial transcriptomics and scRNA-seq identifies spatiotemporally-regulated cell clusters after $\mathrm{MI}$}

To know the specific positional information of these cardiomyocyte clusters seen in our scRNA-seq analysis, we performed spatial transcriptomic analysis using Visium (10X Genomics). A mean of 1,974.4 spots (1,153-3,154 spots) from seven murine hearts (sham, $n=1$; day $1, n=2$; day $7, n=2$; day 14, $n=2$ ) were sequenced and a median of 3,627 genes per spot (interquartile range 2,073-5,425) were detected. Fig. 2a showed both hematoxylin and eosin (H\&E) staining of the heart at each time point after MI surgery and spot region clusters revealed by clustering analysis of Visium data (Fig. 2b). Although the size of infarct regions was slightly different among mice, the characteristic of each cluster's distribution was very similar and reproducible (Extended Data Fig. 1a). We also performed differential gene expression and gene ontology enrichment analysis for each cluster (Fig. 2c, d). Spot regions of cluster Green, characteristic for genes involved in mitochondrial functional pathway, occupied the majority of sham heart and RZ of infarcted hearts. Cluster Brown, characterized by expression of collagens and extracellular matrix genes, was specifically localized in IZ (Fig. 2a, c, d). Spot regions of cluster Red were mainly seen in BZ of the infarcted hearts (Fig. 2a). Almost all marker genes of cluster Red were shared with those of cluster 7 in single-cardiomyocyte RNA-seq analysis (Fig. 1d, e, 2c, d). Genes encoding mechano-sensing proteins such as Csrp3 and Ankrd1 were highly expressed in cluster Red, which were uniquely localized in BZ of the infarcted hearts in acute phase of MI (Fig. 2e). Spatially-regulated expression of these genes in cardiomyocytes was validated by RNA in situ hybridization (Extended Data 
Fig. 1b-e). Subsequently, we determined cell types composing each spot region by integrative analysis of scRNA-seq data from both cardiomyocytes and non-cardiomyocytes (Extended Data Fig. 2) and Visium data. Cardiomyocytes occupied the majority of the spot regions of cluster Green and Red, whereas cardiac fibroblasts accounted a large part of cluster Brown (Fig. 2f).

\section{Weighted gene co-expression network analysis of spatial transcriptomics reveals mechano-sensing genes as a distinct key factor expressed in BZ after MI}

To clarify the transcriptional network of BZ, we performed weighted gene co-expression network analysis (WGCNA) on the Visium data. WGCNA identified nine gene modules (Fig. 3a, Extended Data Fig. 3) and each module contained 40-954 genes. Among nine modules, expressions of Module 1(M1) genes encoding collagens and extracellular matrix proteins were up-regulated selectively in IZ especially in pMI day 7 (Extended Data Fig. 3). The M2 genes were widely seen in the sham hearts and RZ of the infarcted hearts (Extended Data Fig. 3). By contrast, the M3 genes were specifically expressed in IZ of pMI day 1 hearts (Extended Data Fig. 3). Expression patterns of M8 genes were unique and up-regulated only in BZ of the pMI day1 hearts (Fig. 3b). WGCNA of M8 identified mechano-sensing genes such as Csrp3 and Ankrd1 as its central genes (Fig. 3c, d). M8 genes were highly overlapped with the marker genes of cluster Red (Extended Data Fig. 4). Furthermore, single-cardiomyocyte RNA-seq analysis revealed that the cardiomyocytes with lower Csrp3 expression had high expression levels of p53 signaling associated genes such as Cdkn1a, Bax and Fas (Fig. 3e). p53 signaling in cardiomyocytes was shown to induce heart failure both after $\mathrm{Ml}$ and after pressure overload ${ }^{14-16}$, suggesting that Csrp3 in cardiomyocytes adaptively functions against the induction of failing cardiomyocytes.

\section{Csrp3 expressed in cardiomyocytes of BZ adaptively prevents adverse LV remodeling after MI}

To clarify the roles of the mechano-sensing genes specifically upregulated in BZ of the pMI day 1 heart, we generated knockdown AAV9 vectors to deliver shRNA against Csrp3, which was identified as one of the key genes in M8 (Fig. 3c). We injected the knock-down vectors (Extended Data Fig. 5a) into mice through their tail veins two weeks before MI surgery and performed transthoracic echocardiography to analyse their cardiac function (Fig. 4a). The delivered shRNA vectors significantly reduced expressions of Csrp3 at mRNA and protein levels in the heart (Fig. 4b, c). Compared with controls, the AAV9-shCsrp3 injected mice showed more enlarged left ventricles and reduced LV contraction after MI (Fig. 4d-f). Although there was no statistical significance, the AAV9-sh Csrp3 injected mice also tended to die after MI as compared with control mice (Extended Data Fig. 5b).

\section{Discussion}

In the present study, we comprehensively investigated the spatiotemporal gene expression of the heart after $\mathrm{Ml}$ and identified several gene programs which were precisely regulated in a spatiotemporal manner. It has been well known that LV remodeling after MI including expansion of the BZ causes LV dysfunction and dilatation, resulting in heart failure ${ }^{17,18}$. However, the cellular and molecular mechanisms underlying 
remodeling processes in the BZ remain unclear. Previous studies examined expressions of RNA or proteins using myocardial tissues around the BZ dissected from infarcted hearts and reported that expressions of genes associated with angiogenesis, inflammation, apoptotic response and B-type natriuretic peptide (BNP) were highly up-regulated in the $\mathrm{BZ}^{11,19-21}$. However, it was difficult to precisely define spatiotemporal molecular behaviors after $\mathrm{Ml}$ at the single-cell level due to the ambiguous spatial localization of the BZ and the analysis using bulk tissues. In this study, we combined spatial transcriptomics and single-cell RNA-seq to obtain spatiotemporal expression profiles after $\mathrm{Ml}$ at the single-cell level, and identified the mechano-sensing gene Csrp3, expressed in the BZ at an early phase after $\mathrm{Ml}$, as an adaptive regulator of $\mathrm{LV}$ remodeling. Our integrative datasets provide a publicly available resource to further deepen the understanding of pathogenesis of myocardial infarction.

Our spatial transcriptomic analysis clearly revealed that expressions of mechano-sensing genes were upregulated in an acute phase after $\mathrm{MI}$ in the BZ. In a recent study using echocardiography for a porcine MI model, the end-diastolic mean wall stress and myocardial stiffness of the BZ lineally increased throughout 28 days after $\mathrm{Ml}^{22}$, suggesting that cardiomyocytes activate gene expression in response to mechanical stresses during the very acute phase of MI. Csrp3 (MLP) has been reported to be a mechanostress sensor localized at the $Z$ disc $^{23,24}$ and its expression in cardiomyocytes is upregulated by mechanical stretch ${ }^{25}$ as well as pressure overload on the heart ${ }^{16,26}$. It has been reported that Csrp3 induces expression of cardiac hypertrophic genes such as Nppb, Acta 1 and Myh7 in response to pressure overload and phenylephrine stimulation through the activation of calcineurin/NFAT pathway $23,27,28$. Rcan 1, whose expression is correlated with the activity of calcineurin/NFAT pathway ${ }^{29}$, was also upregulated in the cardiomyocytes with high expression levels of Csrp3 in our study (Fig. 3e). By contrast, DNA damage and p53 signaling pathway, an inducer of heart failure ${ }^{14-16}$, was activated in cardiomyocytes with low expression levels of Csrp3 (Fig. 3e), suggesting that activation of Csrp3 in the BZ after MI inhibits DNA damage and p53 signaling activation to prevent the induction of failing cardiomyocytes. In skeletal muscle, Csrp3 has also been reported to regulate autophagy and protect against muscular dystrophy ${ }^{30,31}$. Since autophagy is one of the important cardioprotective mechanisms during post-infarction cardiac remodeling ${ }^{32-35}$, Csrp3 may also protect against adverse cardiac remodeling through the regulation of autophagy. In this study, mice with reduced levels of Csrp3 showed more LV dilatation and dysfunction, indicating exacerbation of LV remodeling after MI. These results suggest that the upregulation of Csrp3 as an acute response to mechanical stress in the BZ soon after MI is important to inhibit LV remodeling and may be a novel target of heart failure.

\section{Methods}

\section{Animal models}

All the animal experiments were approved by the University of Tokyo Ethics Committee for Animal Experiments and strictly adhered to the guidelines for animal experiments of the University of Tokyo (Approved Number P14-106). All wild type C57BL/6 male mice were purchased from CLEA JAPAN. 
All mice were housed in separate cages at a maximum of 6 mice per cage in a specific-pathogen-free, temperature-controlled vivarium under a 12-h light/dark cycle with ad libitum access to food and water.

\section{Operation of myocardial infarction model and echocardiography}

Myocardial infarction (MI) was induced as previously described ${ }^{36}$. Age of mice operated and used for experiments were 9 week old male mice. Mice were anaesthetized by inhalation of $2 \%$ isoflurane. MI was induced by ligation of the left anterior descending artery. Mice that failed to develop MI or died within one day after the operation were excluded from the analysis. Transthoracic echocardiography was performed on conscious mice, using a Vevo 2100 imaging system (Visualsonics, Inc.). To minimize variation in the data, cardiac function was assessed only when the heart rate was $600-700$ beats per minute. M-mode echocardiographic images were obtained from a longitudinal view to measure the size and function of the left ventricle.

\section{Single-cardiomyocyte RNA-seq analysis of mouse samples}

Cardiomyocytes were isolated using Langendorff perfusion from the left ventricle as previously described ${ }^{16}$. Enzymatic dissociation using Langendorff perfusion was performed with $35 \mathrm{~mL}$ enzyme solution (type 2 collagenase $1 \mathrm{mg} / \mathrm{mL}$ [Worthington], protease type XIV $0.05 \mathrm{mg} / \mathrm{mL}$ [Sigma-Aldrich], $\mathrm{NaCl}$ $130 \mathrm{mM}, \mathrm{KCl} 5.4 \mathrm{mM}, \mathrm{MgCl}_{2} 0.5 \mathrm{mM}, \mathrm{NaH}_{2} \mathrm{PO}_{4} 0.33 \mathrm{mM}$, D-glucose $22 \mathrm{mM}$, HEPES $25 \mathrm{mM}$; pH 7.4) preheated to $37^{\circ} \mathrm{C}$, at a rate of $3 \mathrm{~mL} / \mathrm{min}$. To neutralize enzymatic activity, fetal bovine serum (FBS) was added to the solution to a final concentration of $0.2 \%(\mathrm{v} / \mathrm{v})$. After perfusion of digestion buffer, left ventricle was cut according to each sectional region (IR, RR) and cardiomyocytes were subsequently isolated. Cell suspensions were filtered through a 100- $\mu \mathrm{m}$ nylon mesh cell strainer and centrifuged at 100 $\mathrm{g}$ for $2 \mathrm{~min}$, after which the supernatant was discarded. To prevent hypercontraction, the cardiomyocytes were resuspended in medium ( $\mathrm{NaCl} 130 \mathrm{mM}, \mathrm{KCl} 5.4 \mathrm{mM}, \mathrm{MgCl}_{2} 0.5 \mathrm{mM}, \mathrm{NaH}_{2} \mathrm{PO}_{4} 0.33 \mathrm{mM}$, D-glucose $22 \mathrm{mM}$, HEPES $25 \mathrm{mM}$, FBS $0.2 \%$; pH 7.4) containing a low concentration of calcium (0.1 mM). Rodshaped live cardiomyocytes (viability of cardiomyocytes was $\geq 80 \%$ for all time points) were collected immediately with a $0.2-2-\mu \mathrm{L}$ pipette (sample volume, $0.5 \mu \mathrm{L}$ ) and incubated in lysis buffer.

Single-cardiomyocyte cDNA libraries were generated using the Smart-seq2 protocol $^{37}$ and the efficiency of reverse transcription was assessed by examining the cycle threshold (Ct) values of control genes (Tnnt2) from quantitative real-time polymerase chain reaction (qRT-PCR) using a CFX96 Real-Time PCR Detection System (Bio-Rad), and the distribution of cDNA fragment lengths was assessed using LabChip GX (Perkin Elmer) and/or TapeStation 2200 (Agilent Technologies). The following primer sets were used for qRT-PCR: Tnnt2 mRNA forward: TCCTGGCAGA GAGGAGGAAG; Tnnt2 mRNA reverse: TGCAGGTCGA ACTTCTCAGC. A Ct value of 25 was set as the threshold. The sequencing libraries were subjected to paired-end 51-bp RNA sequencing on a HiSeq 2500 (Illumina) in rapid mode.

For the isolation and collection of non-cardiomyocytes, hearts were minced and enzymatically dissociated using $2 \mathrm{mg} / \mathrm{mL}$ type 2 collagenase (Worthington), $1 \mathrm{mg} / \mathrm{mL}$ dispase (Roche), and $20 \mathrm{U} / \mathrm{mL}$ 
DNase I (Roche), with 5 cycles of digestion for a total $40 \mathrm{~min}$ at $37^{\circ} \mathrm{C}$. After using a $40-\mu \mathrm{m}$ cell strainer (Greiner) to remove the cardiomyocytes, 5,000 cells were prepared to a concentration of $1000 \mathrm{cells} / \mu \mathrm{L}$ and loaded into the Chromium Controller and single-cell cDNA libraries were generated using the Chromium 3' v3 chemistry kit (10x Genomics) according to the manufacturer's instruction. Libraries were sequenced on a NovaSeq 6000 System (Illumina) using a NovaSeq S4 Reagent Kit (200 cycles, 20027466, Illumina).

\section{Single-cardiomyocyte RNA-seq data processing}

Raw sequencing reads from single-cardiomyocyte RNA sequencing libraries were trimmed to remove adapter sequences and low-quality bases using fastp-0.20.0 ${ }^{38}$ with the parameters "--cut_tail -cut_tail_window_size 1 -cut_tail_mean_quality 30 -length_required 30 ". The reference transcript data and the gene annotation file for mouse was downloaded from GENCODE (release 23 , https://www.gencodegenes.org/ $)^{39}$. The clean reads were aligned to the mouse genome $(\mathrm{mm} 10)$ using STAR (version 2.7.2b) ${ }^{40}$. The reads aligned to exon were counted using featureCounts ${ }^{41}$. TPM normalization was calculated with reads mapped to the nuclear genome. We removed the low-quality cardiomyocytes with less than 2500 detected genes, which were used for the downstream analysis.

\section{Identification of cell clusters and DEGs in single-cardiomyocyte RNA-seq}

$\log _{2}(T P M+1)$ counts were used as the input of Seurat R package (version 4.0.0) ${ }^{42}$. Each dataset was merged, and the merged data were used for dimensionality reduction and cluster detection. Differentially expressed genes (DEGs) were detected by using FindMarkers in Seurat $(\log 2 \mathrm{fc}$.threshold $>0.25$ and p_val_adj < 0.05). Top DEGs were presented according to the $\log _{2}$ fold-change of the average expression (avg.log2FC). Finally, GO enrichment analysis was performed using Metascape with GO biological processes $^{43}$.

\section{Spatial transcriptome analysis of mouse MI model}

Frozen samples were embedded in OCT (TissueTek Sakura) and cryosectioned at $-14^{\circ} \mathrm{C}$ (Leica CM1860). Sections were placed on chilled Visium Spatial Gene Expression Slides (2000233, 10X Genomics), and adhered by warming the back of the slide. Tissue sections were then fixed in chilled methanol and stained according to the Visium Spatial Gene Expression User Guide (CG000239 Rev D, 10X Genomics). For gene expression samples, tissue was permeabilized for 15 minutes, which was selected as the optimal time based on tissue optimization time course experiments (CG000238 Rev D, 10X Genomics). Brightfield histology images of Hematoxylin and Eosin stain were taken using a 10X objective on a BZX700 microscope (Keyence Corporation, Itasca, Illinois). Raw images were stitched together using BZ-X analyzer software (Keyence Corporation) and exported as TIFF files.

Libraries were prepared according to the Visium Spatial Gene Expression User Guide (CG000239 Rev D, 10X Genomics) and sequenced on a NovaSeq 6000 System (Illumina) using a NovaSeq S4 Reagent Kit (200 cycles, 20027466, Illumina), at a sequencing depth of approximately 250-400M read-pairs per 
sample. Sequencing was performed using the following read protocol: read 1, 28 cycles; i7 index read, 10 cycles; i5 index read, 10 cycles; read 2, 91 cycles.

\section{Spatial transcriptome data processing}

Raw FASTQ files and histology images were processed by sample with the Space Ranger software (ver 1.2.1, 10X Genomics), against the Cell Ranger mm10 reference genome "refdata-gex-mm10-2020-A", available at: https://cf.10xgenomics.com/supp/spatial-exp/refdata-gex-mm10-2020-A.tar.gz. Raw counts were used as the input of Seurat R package (version 4.0.0), log normalization was implemented separately for each dataset and integrated them. The integrated data were used for dimensionality reduction and cluster detection. Differentially expressed genes were detected by using FindMarkers in Seurat (log2fc.threshold $>0.25$ and p_val_adj < 0.05). Top DEGs were presented according to the log2 fold-change of the average expression (avg.log2FC). Finally, GO enrichment analysis was performed using Metascape with GO biological processes ${ }^{43}$.

\section{Weighted gene co-expression analysis}

CPM (counts per million) counts were used as the input of WGCNA analysis (version 1.69) ${ }^{44}$. All genes expressed at more than 700 spots were used for analysis. The soft power threshold was analyzed with the "pickSoftThreshold" function and was applied to construct a signed network and calculate module eigengene expression using the "blockwiseModules" function. Modules with $<30$ genes were merged to their closest larger neighboring module. To visualize the weighted co-expression networks, Cytoscape (version 3.8.0) ${ }^{45}$ with the "prefuse force-directed layout" was used. Signed eigengene-based connectivity of a gene in a module reflected node size.

\section{AAV9-sh Csrp3 infection}

The AAV vectors were prepared by VectorBuilder Inc. (https://en.vectorbuilder.com) according to established procedures $^{46}$. Briefly, AAV vectors of serotypes 2 and 9 were generated in HEK293T cells, using triple-plasmid co-transfection for packaging. Viral stocks were obtained by $\mathrm{CsCl} 2$-gradient centrifugation. Titration of AAV viral particles was performed by real-time PCR quantification of the number of viral genomes, measured as CMV copy number. The viral preparations had a titer between $1 \times 10^{12}$ and $5 \times 10^{12}$ genome copy (GC)/mL. Viruses were administered in $100-\mu \mathrm{L}$ saline via tail-vein injection. $3 \times 10^{11} \mathrm{GC}$ doses of AAV9-eGFP or $3 \times 10^{11} \mathrm{GC}$ doses of AAV9-sh Csrp3 were administered to the uninjured mice 2 week before MI surgery.

\section{RNA in situ hybridization}

For RNA fluorescent in situ hybridization, the RNAscope system (Advanced Cell Diagnostics) was used with a probe against murine $C s r p 3$ and Tnnt2 mRNA as previously described ${ }^{47}$. Frozen sections $(10 \mu \mathrm{m})$ were fixed in PBS containing 4\% paraformaldehyde for $5 \mathrm{~min}$ at room temperature, dehydrated by serial immersion in $50 \%, 70 \%$, and $100 \%$ ethanol for 5 min at room temperature, and treated with protease for 
30 min at room temperature. The probe was then hybridized for $2 \mathrm{~h}$ at $40^{\circ} \mathrm{C}$, followed by RNAscope amplification, and co-stained with DAPI to detect nuclei. Images were obtained using each 20X or 40X objective on a BZ-X700 microscope (Keyence Corporation, Itasca, Illinois).

\section{Tissue histology}

For histological analysis, mice were anaesthetized by isoflurane inhalation and sacrificed by cervical dislocation. The chest was opened, and the heart was flushed with cold PBS via cardiac apical insertion of a 25-gauge needle. The right atrium was cut to allow drainage of blood from the heart, and the mice were briefly perfused with cold fixative (4\% paraformaldehyde in PBS) through the apex of the heart. Tissues were excised, flushed with fixative, incubated in fixative for $12 \mathrm{~h}$ at $4{ }^{\circ} \mathrm{C}$ with gentle rotation, and finally embedded in paraffin. Paraffin-embedded heart tissues were sectioned into $4-\mu \mathrm{m}$ slices using an SM2010 R Sliding Microtome (Leica Biosystems), and sections were stained with Picrosirius Red/Fast Green dyes. Brightfield histology images were taken using a 10X objective on a BZ-X700 microscope (Keyence Corporation, Itasca, Illinois).

\section{qRT-PCR}

Expression of Csrp3 mRNA was evaluated by qRT-PCR using a CFX96 Real-Time PCR Detection System, and the relative expression levels of the target genes were normalized to the expression of an internal control gene, using the comparative Ct method. The following primer sets were used for qRT-PCR.

Rps18mRNA forward, CTTAGAGGGACAAGTGGCG

Rps18 mRNA reverse, ACGCTGAGCCAGTCAGTGTA

Csrp3 mRNA forward, TGAGAAGGTCATGGGAGGTG

Csrp3 mRNA reverse, CTTGCTGTGTAAGCCCTCCA

\section{Western blotting}

Heart tissue was homogenized and lysed with RIPA buffer containing $10 \mathrm{mM}$ Tris- $\mathrm{HCl}, 150 \mathrm{mM} \mathrm{NaCl}, 5$ mM EDTA, $1 \%$ Triton X-100, $1 \%$ sodium deoxycholate, and $0.1 \%$ sodium dodecyl sulfate with protease and phosphatase inhibitor cocktails for $30 \mathrm{~min}$ on ice. Lysates were centrifuged at $15,000 \times \mathrm{g}$ for $30 \mathrm{~min}$ and the supernatants were used as whole-cell extracts. Total protein concentrations in the supernatants were measured using a bicinchoninic acid assay (Pierce BCA Protein Assay Kit; Thermo Scientific). For immunoblot analysis, extracted protein samples were separated on $5 \%-20 \%$ Mini-PROTEAN TGX precast gradient gels (Bio-Rad) and transferred onto nitrocellulose membranes (BioRad). Membranes were blocked with $5 \%$ FBS in Tris-buffered saline plus $0.05 \%$ Tween and incubated overnight at $4{ }^{\circ} \mathrm{C}$ with primary antibodies, followed by horseradish peroxidase-conjugated secondary antibodies and ECL Plus (GE Healthcare). Immunoreactive signals were detected using a LAS 4000 analyzer (GE Healthcare). The following antibodies were used for western blotting: anti-Csrp3 monoclonal antibody (ab173301, Abcam, 
1:2000), HRP-linked horse anti-rabbit IgG antibody (\#7074, Cell Signaling Technology, 1:3000), HRP-linked horse anti-mouse IgG antibody (\#7076, Cell Signaling Technology, 1:3000) and anti-Actin monoclonal Antibody (\#MA5-11869, Invitrogen, 1:5000).

\section{Declarations}

\section{Data Availability}

The authors declare that all data are available in the article file and Extended Data information files or available from the authors upon reasonable request. The sequencing and alignment metrics of singlecardiomyocyte RNA-seq and Visium are provided as Extended data tables. The source data underlying Figures. 1e, 2c, d, 3c, d, 4b, c, f and Extended Data Fig. 3b are provided as data source tables. Singlecardiomyocyte, single-cell RNA sequencing and spatial transcriptomic data have been deposited in GSE176092 (https://www.ncbi.nlm.nih.gov/geo/query/acc.cgi?acc=GSE176092). The secure token is "ersbeoycldebpgd").

\section{Acknowledgements}

We thank K. Shiina for next-generation sequencing support and Y. Yokota and N. Furukawa for experimental support. This work was supported by grants from a Grant-in-Aid for Young Scientists (to T.K., M.K. and M.I.), the Japan Foundation for Applied Enzymology (to S.N. and T.K.), the SENSHIN Medical Research Foundation (to S.N. and T.K.), the Kanae Foundation for the Promotion of Medical Science (to S.N.), MSD Life Science Foundation (to S.N. and T.K.), The Tokyo Biomedical Research Foundation (to S.N.), Astellas Foundation for Research on Metabolic Disorders (to S.N.), The Novartis Foundation (Japan) for the Promotion of Science (to S.N.), the Japanese Circulation Society (to S.N.), the Takeda Science Foundation (to S.N.), The Cell Science Research Foundation (to S.N.), a Grant-in-Aid for Scientific Research (B) (to S.N.), a Grant-in-Aid for Scientific Research (A) (to I.K.), and AMED (JP21ek0210152, JP21 gm6210010, JP20bm0704026, JP21ek0210141, JP21ek0109440, JP21ek0109487, JP21gm0810013, JP21 km0405209, JP21 ek0210118, JP21ek0109406, JP21ek0109543) (to S.N., H.A., and I.K.).

\section{Author contributions}

S.Y., T.K., S.N., H.A., and I.K. conceived the project, designed the study, and interpreted the results; S.Y., B.Z. and T.K. collected single cells and generated the single-cell sequencing data; S.Y. and S.H. performed computational analyses; H.A. provided support for computational analyses; T.K., B.Z, and S.Y. performed MI procedure, conducted functional analysis, performed biochemical experiments, and analyzed the data; B.Z., Z.D., S.I., K.S., T.Y., T.S., M.K., K.F., M.K., M.I., M.H., H.T., N.T., H.M., H.A., and I.K. provided experimental and analytical support; S.Y., T.K., S.H., S.N., H.A., and I.K. wrote the manuscript with input from all authors.

\section{Competing Interests}

There are no competing interests and the authors declare no competing interests. 


\section{References}

1. Virani, S. S. et al. Heart disease and stroke statistics-2020 update: A report from the American Heart Association. Circulation (2020). doi:10.1161/CIR.0000000000000757

2. Prabhakaran, D. et al. Cardiovascular, respiratory, and related disorders: key messages from Disease Control Priorities, 3rd edition. Lancet 391, 1224-1236 (2018).

3. Reiner, Ž. Hypertriglyceridaemia and risk of coronary artery disease. Nat. Rev. Cardiol. 14, 401-411 (2017).

4. Cui, M. et al. Dynamic Transcriptional Responses to Injury of Regenerative and Non-regenerative Cardiomyocytes Revealed by Single-Nucleus RNA Sequencing. Dev. Cell 53, 102-116.e8 (2020).

5. Bahit, M. C., Kochar, A. \& Granger, C. B. Post-Myocardial Infarction Heart Failure. JACC Hear. Fail. 6, 179-186 (2018).

6. Leach, J. P. et al. Hippo pathway deficiency reverses systolic heart failure after infarction. Nature 550, 260-264 (2017).

7. Mann, D. L., Bogaev, R. \& Buckberg, G. D. Cardiac remodeling and myocardial recovery: Lost in translation? Eur. J. Heart Fail. 12, 789-796 (2010).

8. Fu, X. et al. Specialized fibroblast differentiated states underlie scar formation in the infarcted mouse heart. J. Clin. Invest. 128, 2127-2143 (2018).

9. Vagnozzi, R. J. et al. An acute immune response underlies the benefit of cardiac stem cell therapy. Nature 577, 405-409 (2020).

10. Martini, E. et al. Single-Cell Sequencing of Mouse Heart Immune Infiltrate in Pressure OverloadDriven Heart Failure Reveals Extent of Immune Activation. Circulation 140, 2089-2107 (2019).

11. Van Duijvenboden, K. et al. Conserved NPPB + Border Zone Switches from MEF2- to AP-1-Driven Gene Program. Circulation 140, 864-879 (2019).

12. Ounzain, S. et al. Genome-wide profiling of the cardiac transcriptome after myocardial infarction identifies novel heart-specific long non-coding RNAs. Eur. Heart J. 36, 353-368 (2015).

13. Lacraz, G. P. A. et al. Tomo-Seq Identifies SOX9 as a Key Regulator of Cardiac Fibrosis during Ischemic Injury. Circulation 136, 1396-1409 (2017).

14. Shukla, P. C. et al. BRCA1 is an essential regulator of heart function and survival following myocardial infarction. Nat. Commun. 2, 511-593 (2011). 
15. Sano, M. et al. p53-induced inhibition of Hif-1 causes cardiac dysfunction during pressure overload. Nature 446, 444-448 (2007).

16. Nomura, S. et al. Cardiomyocyte gene programs encoding morphological and functional signatures in cardiac hypertrophy and failure. Nat. Commun. 9, 4435 (2018).

17. Tada, Y. et al. Myocardial viability of the peri-infarct region measured by T1 mapping post manganese-enhanced MRI correlates with LV dysfunction. Int. J. Cardiol. 281, 8-14 (2019).

18. Yan, A. T. et al. Characterization of the peri-infarct zone by contrast-enhanced cardiac magnetic resonance imaging is a powerful predictor of post-myocardial infarction mortality. Circulation 114, 32-39 (2006).

19. Yang, L. et al. Quantitative Proteomics and Immunohistochemistry Reveal Insights into Cellular and Molecular Processes in the Infarct Border Zone One Month after Myocardial Infarction. J. Proteome Res. 16, 2101-2112 (2017).

20. Meng, Q. et al. Expression profiles of long noncoding RNAs and messenger RNAs in the border zone of myocardial infarction in rats. Cell. Mol. Biol. Lett. 24, 1-13 (2019).

21. Zimmermann, M. et al. Analysis of region specific gene expression patterns in the heart and systemic responses after experimental myocardial ischemia. Oncotarget 8, 60809-60825 (2017).

22. Torres, W. M. et al. Regional and temporal changes in left ventricular strain and stiffness in a porcine model of myocardial infarction. Am. J. Physiol. - Hear. Circ. Physiol. 315, H958-H967 (2018).

23. Knöll, R. et al. The cardiac mechanical stretch sensor machinery involves a $Z$ disc complex that is defective in a subset of human dilated cardiomyopathy. Cell 111, 943-955 (2002).

24. Makarewich, C. A. et al. The DWORF micropeptide enhances contractility and prevents heart failure in a mouse model of dilated cardiomyopathy. Elife 7, 1340-1342 (2018).

25. Kuhn, C. et al. Cardiac remodeling is not modulated by overexpression of muscle LIM protein (MLP). Basic Res. Cardiol. 107, (2012).

26. Buyandelger, B. et al. MLP (muscle LIM protein) as a stress sensor in the heart. Pflugers Arch. Eur. J. Physiol. 462, 135-142 (2011).

27. Heineke, J. et al. Attenuation of cardiac remodeling after myocardial infarction by muscle LIM protein-calcineurin signaling at the sarcomeric Z-disc. Proc. Natl. Acad. Sci. U. S. A. 102, 1655-1660 (2005).

28. Boateng, S. Y., Senyo, S. E., Qi, L., Goldspink, P. H. \& Russell, B. Myocyte remodeling in response to hypertrophic stimuli requires nucleocytoplasmic shuttling of muscle LIM protein. J. Mol. Cell. Cardiol. 47, 
426-435 (2009).

29. Crabtree, G. R. \& Olson, E. N. NFAT signaling: Choreographing the social lives of cells. Cell 109, 6779 (2002).

30. Rashid, M. M. et al. Muscle LIM protein/CSRP3: a mechanosensor with a role in autophagy. Cell Death Discov. 1, 1-12 (2015).

31. Cui, C. et al. The autophagy regulatory molecule CSRP3 interacts with LC3 and protects against muscular dystrophy. Int. J. Mol. Sci. 21, (2020).

32. Liu, C. Y. et al. LncRNA CAIF inhibits autophagy and attenuates myocardial infarction by blocking p53-mediated myocardin transcription. Nat. Commun. 9, (2018).

33. Wu, D., Zhang, K. \& Hu, P. The role of autophagy in acute myocardial infarction. Front. Pharmacol. 10, (2019).

34. Sciarretta, S. et al. Trehalose-Induced Activation of Autophagy Improves Cardiac Remodeling After Myocardial Infarction. J. Am. Coll. Cardiol. 71, 1999-2010 (2018).

35. Kanamori, H. et al. The role of autophagy emerging in postinfarction cardiac remodeling. Cardiovasc. Res. 91, 330-339 (2011).

36. Toko, H. et al. ATF6 is important under both pathological and physiological states in the heart. J. Mol. Cell. Cardiol. 49, 113-120 (2010).

37. Picelli, S. et al. Full-length RNA-seq from single cells using Smart-seq2. Nat. Protoc. 9, 171-181 (2014).

38. Chen, S., Zhou, Y., Chen, Y. \& Gu, J. Fastp: An ultra-fast all-in-one FASTQ preprocessor. Bioinformatics 34, i884-i890 (2018).

39. Frankish, A. et al. GENCODE reference annotation for the human and mouse genomes. Nucleic Acids Res. 47, D766-D773 (2019).

40. Dobin, A. et al. STAR: Ultrafast universal RNA-seq aligner. Bioinformatics 29, 15-21 (2013).

41. Liao, Y., Smyth, G. K. \& Shi, W. FeatureCounts: An efficient general purpose program for assigning sequence reads to genomic features. Bioinformatics 30, 923-930 (2014).

42. Hao, Y. et al. Integrated analysis of multimodal single-cell data. Cell 184, 1-15 (2021).

43. Zhou, Y. et al. Metascape provides a biologist-oriented resource for the analysis of systems-level datasets. Nat. Commun. 10, (2019). 
44. Langfelder, P. \& Horvath, S. WGCNA: An R package for weighted correlation network analysis. BMC Bioinformatics 9, 559 (2008).

45. Paul Shannon, 1 et al. Cytoscape: A Software Environment for Integrated Models. Genome Res. 13, 2498-2504 (2003).

46. Ruozi, G. et al. AAV-mediated in vivo functional selection of tissue-protective factors against ischaemia. Nat. Commun. 6, 7388 (2015).

47. Satoh, M. et al. High-throughput single-molecule RNA imaging analysis reveals heterogeneous responses of cardiomyocytes to hemodynamic overload. J. Mol. Cell. Cardiol. 128, 77-89 (2019).

Figures 


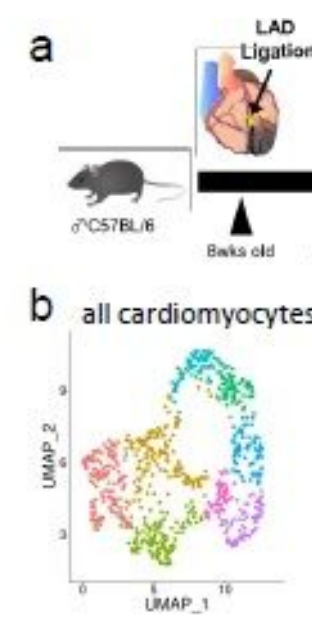

LAD
Ligatio

SCRNA-seg
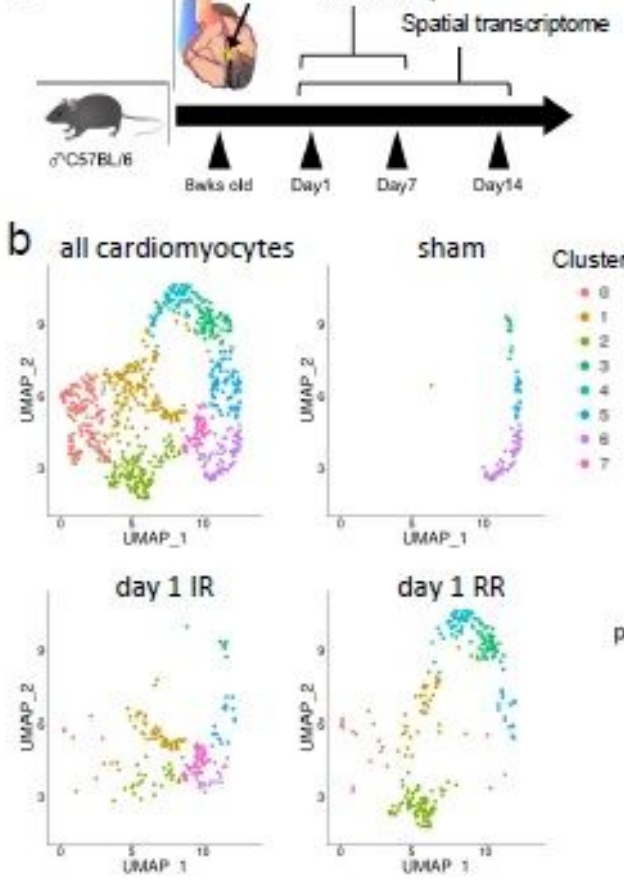

day 7 IR

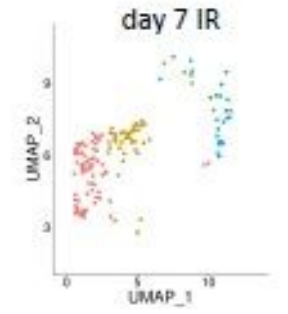

C

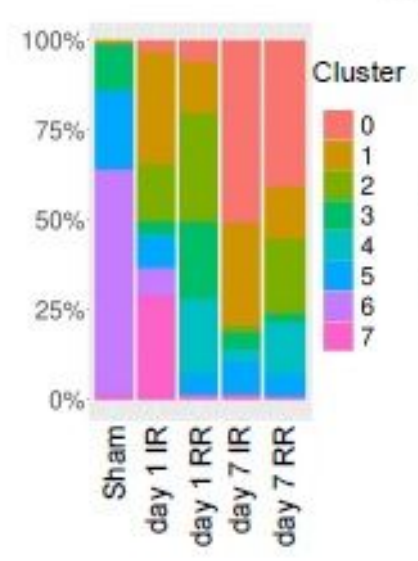

day 7 RR

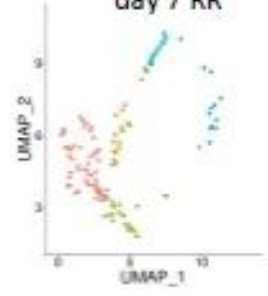

d e

Cluster 0

extracellular matrix organization

blood vessel development

collagen metabolic process
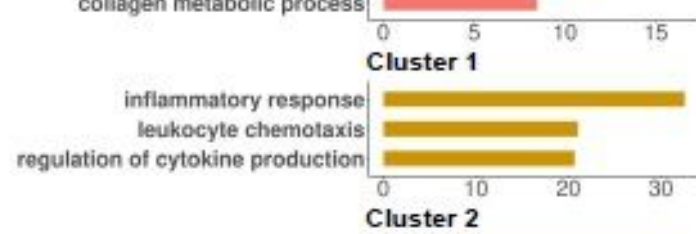

blood vessel morphogenesis

regulation of endothelial cell proliferation

cellular response to growth tactor stumulus

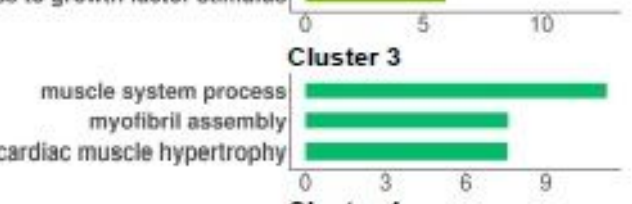

\section{Cluster 4}

positive regulation of NIKNF-kappaB signaling regulation of protein catabolic process ribosome biogenesis

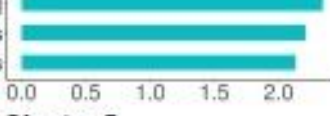

Cluster 5

regulation of hest contraction triglyceride biosynthetic process oxidation-reduction process

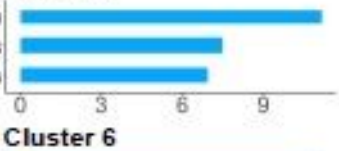

Cluster 6

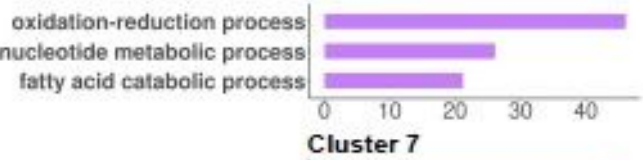

oxidative phosphorylation heart contraction response to muscle stretch $\begin{array}{ccc}10 & 20 \\ -\log _{10}(\mathrm{P} \text { value })\end{array}$

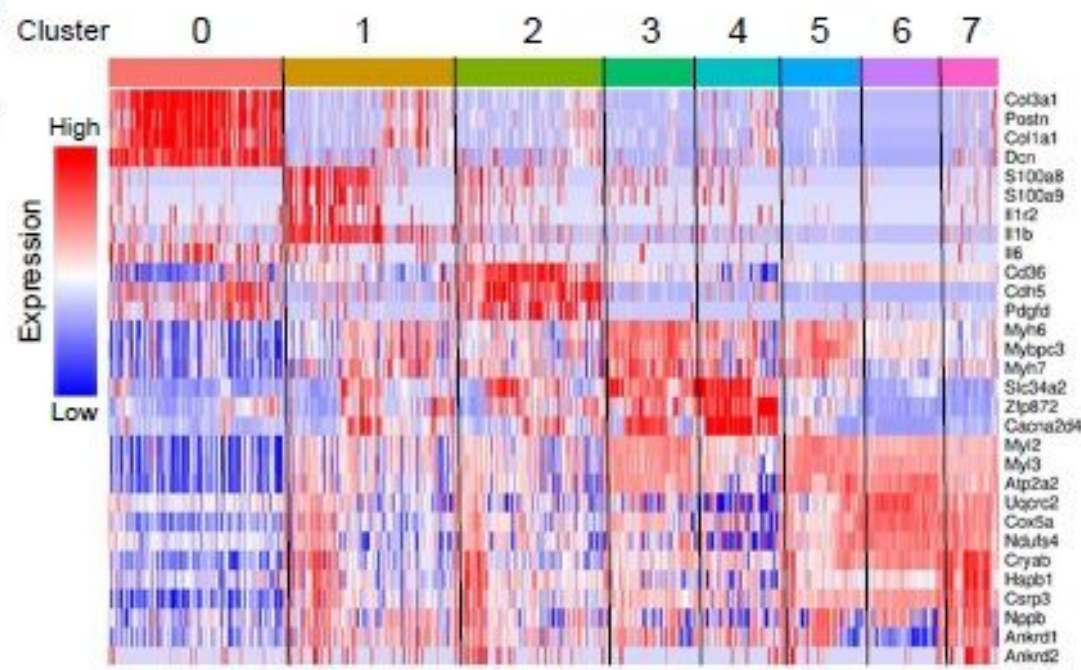

\section{Figure 1}

Single-cardiomyocyte RNA-seq identifies spatiotemporally distinct cell clusters after MI. a, Experimental scheme of single-cell transcriptomic analysis after myocardial infarction (MI). LAD, left anterior descending artery. b, The Uniform Manifold Approximation and Projection (UMAP) plot of all cardiomyocytes and cardiomyocytes derived from 5 conditions (Sham, post MI day 1 ischemic regions (pMI day $1 \mathrm{IR}$ ), post MI day 1 remote regions (pMI day $1 \mathrm{RR}$ ), post MI day 7 ischemic regions (pMI day 7 
$\mathrm{IR})$, and post MI day 7 remote regions (pMI day $7 \mathrm{RR})$ ). All cardiomyocytes were classified into eight cell clusters (Cluster 0-7). Each cell (dot) was colored by cell clusters. c, Bar plot showing the distribution of cell clusters at each time point and region. $d$, Gene expression heatmap showing the representative differentially expressed genes in each cell cluster. e, Bar plot showing gene ontology enrichment of top 100 marker genes or all marker genes if less than 100 in each cell cluster.

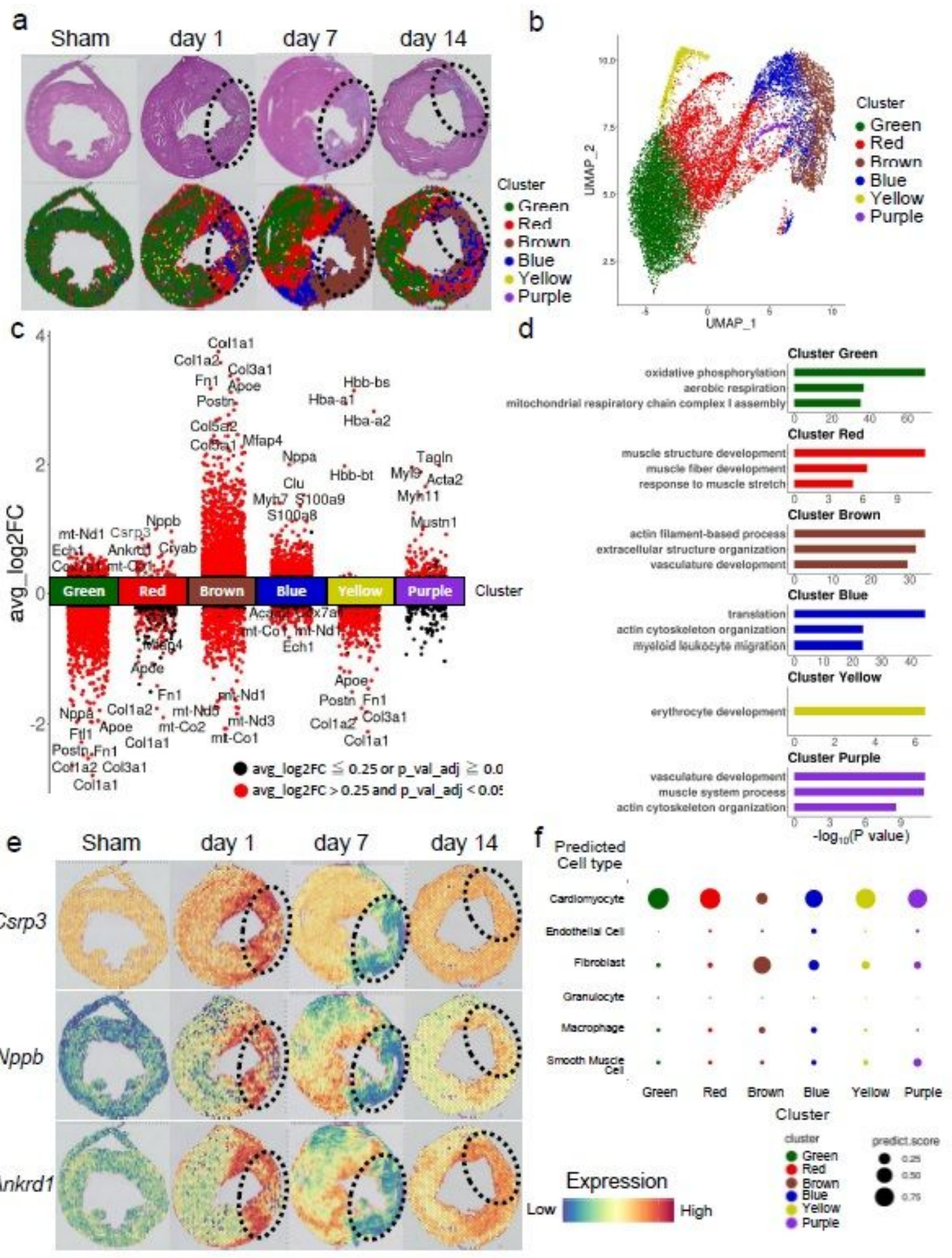

Figure 2 
Integrative analysis of spatial transcriptomics and scRNA-seq identifies spatiotemporally-regulated cell clusters after MI. a, Hematoxylin and eosin (H\&E) staining of whole heart at each time point after MI surgery (above). Clusters found in b. were overlaid on the H\&E images above (below). The areas surrounded by dotted lines indicate the infarcted zone. $b$, UMAP plot of all gene expression spots on the tissue from each time point. All spots (dots) were classified into six clusters (named by the color of dots). c, Gene expression plot according to average-log2 fold change (avg_log2FC) values in each cluster. Each dot represents a single gene. Red dots are differentially expressed genes (avg_log2FC and p_val_adj < 0.05 ) and black dots are the others. Genes that are characteristically up- and down-regulated in each cluster were printed. p_val_adj, adjusted p-value. d, Bar plot showing gene ontology (GO) enrichment in each cluster. e, Visualization of expression level of representative genes in cluster Red at each time point. The areas surrounded by dotted lines indicate the infarcted zone. $\mathrm{f}$, Cell type prediction in each spot guided by single-cell RNA-seq data as a reference. 
a
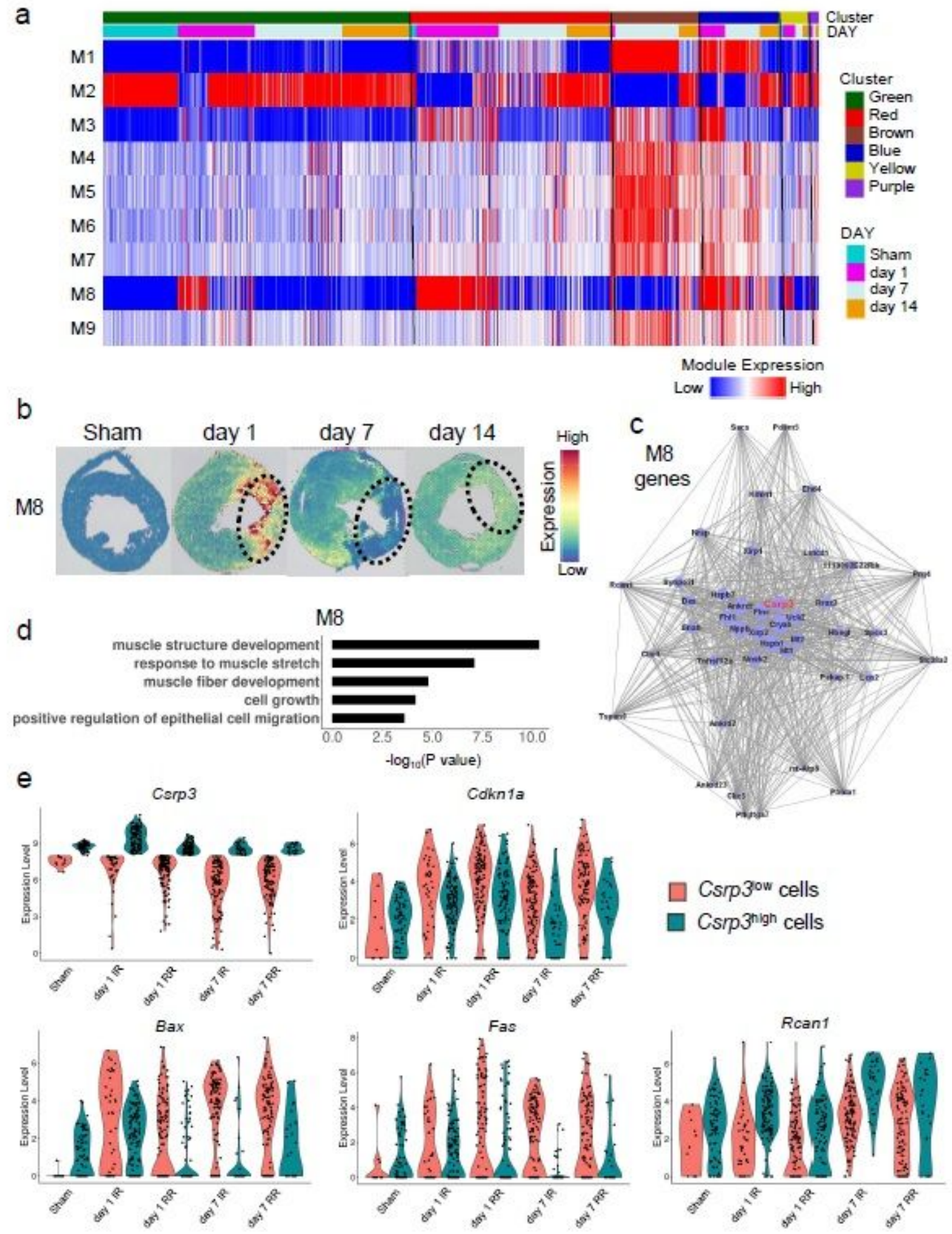

\section{Figure 3}

Weighted gene co-expression network analysis of spatial transcriptomics reveals mechano-sensing genes as a distinct key factor expressed in the BZ after MI. a, Expression heatmap showing mean expression level of each gene module (M1-M9) sorted by clusters found in Fig. 2b. Horizontal axis are colored by Cluster (1st row) and time point after MI (2nd row). b, Visualization of module expression level of a characteristic gene module (module 8; M8) found by weighted gene co-expression network analysis at 
each time point. The areas surrounded by dotted lines indicate infarcted zone. c, Gene network of highly correlated genes in M8. Node size represents signed eigengene-based connectivity of a gene in a module. d, Bar plot showing gene ontology (GO) enrichment of M8. The top five GO terms were listed according to their p-values. e, Violin plot showing gene expressions of Csrp3, Cdkn1a, Bax, Fas and Rcan1 split by Csrp3low cells or Csrp3high cells at each region and time point. Csrp3low cells were defined as $\log 2(T P M+1)$ of $C s r p 3<8$ and Csrp3high cells were the others. IR, ischemic region; RR, remote region.

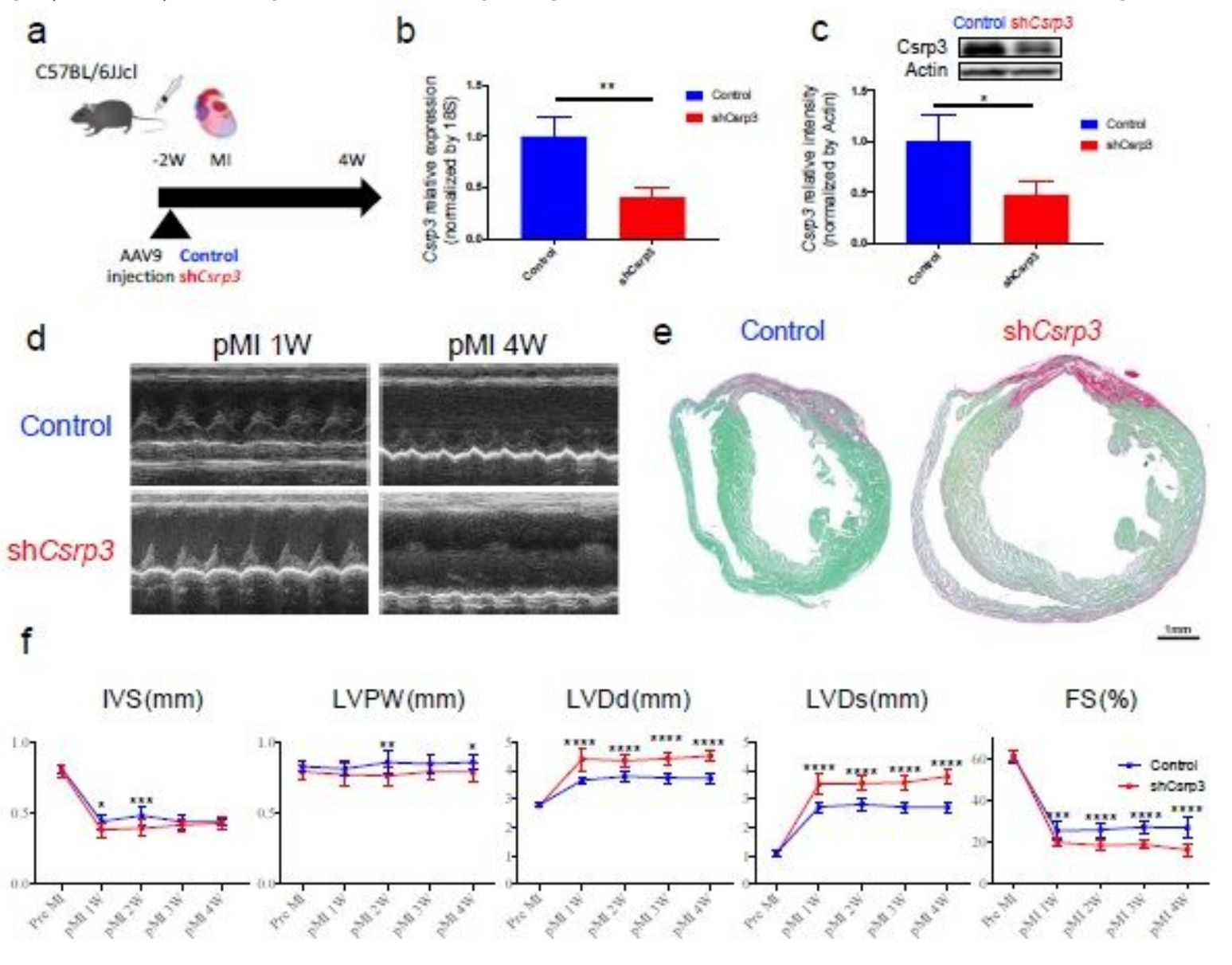

Figure 4

Csrp3 expressed in cardiomyocytes in the BZ adaptively limits adverse LV remodeling after MI. a, Experimental design for testing the effect of AAV9-shCsrp3 vectors. b, mRNA expression levels of Csrp3 in Control and AAV9-shCsrp3 injected hearts were tested 2 weeks after injection using qRT-PCR ( $n=3$ vs 3 ). Data are shown as mean and SD. ** $P<0.01$; Significance was determined by unpaired t-test. $c$, Western blot analysis of Csrp3 using Control and AAV9-shCsrp3 injected hearts were tested 2 weeks after injection $\left(n=4\right.$ vs 4). Data are shown as mean and SD. ${ }^{P}<0.05$; Significance was determined by unpaired t-test. d, Representative echocardiographic images of Control vs AAV9-shCsrp3 injected mice. e, Histochemical detection of collagen fibres by Sirius Red/Fast Green dye staining in Control and AAV9-shCsrp3 injected mice at 1 week after MI surgery. $f$, Echocardiographic assessment of the heart of Control and AAV9shCsrp3 injected mice after MI surgery. Data are shown as mean and SD. $n=12$ (Control) vs 10

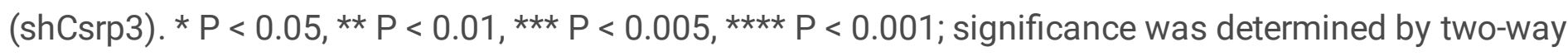
analysis of variance (ANOVA) with Bonferroni's multiple comparison test. 


\section{Supplementary Files}

This is a list of supplementary files associated with this preprint. Click to download.

- 20210612nreditorialpolicychecklist.pdf

- table.xlsx

- 20210612nrreportingsummary.pdf

- ExtendedData.pdf 